\title{
La hipertensión arterial, ¿Sólo un biomarcador de la enfermedad ateroesclerótica sistémica?
}

\author{
OSCAR ROMÁN A. ${ }^{1}$, MARTA BADILLA S. ${ }^{2}$, ANA MARÍA DUSSAUBAT A. ${ }^{1}$
}

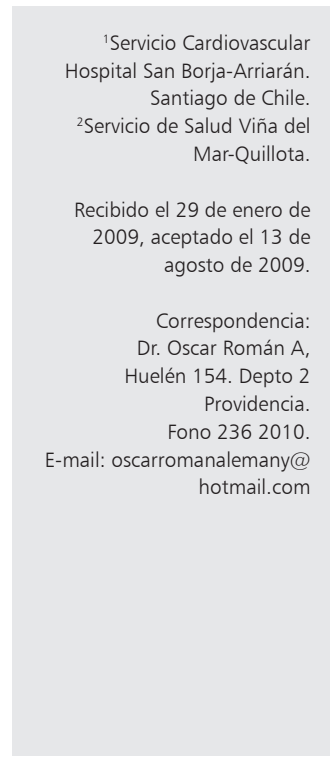

\section{Hypertension as a biological marker of systemic atherosclerotic disease}

There is a close link between hypertension and atherosclerosis. Hypertension causes atherosclerotic damage of several organs, called target organs and the risk factors for hypertension and atherosclerosis are very similar. The risk of mortality associated to hypertension increases with blood pressure values below the cutoff point of normality (140/90 $\mathrm{mm} \mathrm{Hg}$ ) and even below 130/85 $\mathrm{mm} \mathrm{Hg}$ and includes a stage called pre hypertension. Moreover, the initial damage of the arterial walls and target organs are present before there is a significant elevation of blood pressure. Therefore, hypertension could become a biological marker of the evolution of an underlying atherosclerotic process. A new pathophysiological paradigm has been proposed in which the severity of hypertension is not classified according to blood pressure values, but rather on the initiation and progression of vascular damage among target organs. These alterations determine the prognosis and management of systemic vascular damage, that can be called "hypertensive atherosclerotic disease" or simply systemic atherosclerotic disease.

(Rev Med Chile 2010; 138: 346-351).

Key words: Atherosclerosis; Biological markers; Hypertension.

\begin{abstract}
A comienzos de la década 1990-99, en un corte a los 15 años de un seguimiento prospectivo observacional de hipertensos esenciales tratados, nuestro grupo comunicó que los pacientes se complicaban y fallecían por compromiso ateroesclerótico de diversos órganos, siendo el infarto miocárdico, el accidente cerebrovascular, las insuficiencias cardíaca y renal, el aneurisma aórtico y la atero-trombosis de extremidades inferiores los cuadros más relevantes ${ }^{1}$. Tales cuadros patológicos eran y son considerados aún, enfermedades independientes, aunque la arterioesclerosis aparece como su sustrato patogenético, sea de tipo trombótico obstructivo o por ruptura arterial con hemorragia consecutiva. Estas complicaciones aparecían aun cuando se hubiese reducido significativamente o aun normalizado la presión arterial (PA) con el tratamiento
\end{abstract}

farmacológico en uso, de acuerdo a los consensos internacionales vigentes en esa época ${ }^{2-4}$.

Frente a este parcial fracaso clínico surgieron dos hipótesis: a) la reducción de la PA no habría sido suficiente, debiendo optimizarse la terapia para lograr la normotensión; b) que el tratamiento estuviese llegando tarde para evitar o paliar el compromiso ya en marcha de los órganos que se dañaban, que pasaron a llamarse órganos blanco.

\section{Evolución del conocimiento}

La primera hipótesis establecía que la magnitud de la reducción de la PA con el tratamiento farmacológico no era suficiente. $\mathrm{Al}$ amparo de esta suposición, se produjo una sucesiva disminución de la meta o nivel de corte de la normalidad de 
la PA. Así, desde los años 60, se fue reduciendo la meta de PA diastólica desde cifras de $120 \mathrm{mmHg}$, a 114, 110, hasta llegar a $90 \mathrm{mmHg}^{5-7}$. La meta de la PA sistólica también se redujo de 160 a 140 $\mathrm{mmHg}^{3,4}$. Manteniendo estas metas, en 1990 un metaanálisis de 9 estudios con más de 400.000 pacientes seguidos por 6 a 25 años, comprobó la existencia de una correlación directa y significativa entre las cifras de PA sistólica y diastólica con el riesgo de aparición de complicaciones cardiovasculares, especialmente del infarto miocárdico (IAM) y del accidente cerebro-vascular (AVE) ${ }^{8}$. Lo interesante del estudio fue la comprobación que el riesgo de eventos cardiovasculares $(\mathrm{C}-\mathrm{V})$ se mantenía por debajo de los límites de normalidad, de modo que presiones diastólicas de 85 y aun 80 $\mathrm{mmHg}$ presentaban un riesgo evidente, aunque en menor proporción ${ }^{8}$.

De esta observación derivó la indicación de reducir la PA diastólica a cifras inferiores a 90 mmHg. Con este objetivo, en 1998, el estudio HOT demostró que reduciendo el corte de normalidad de la PA diastólica a $85 \mathrm{mmHg}$ disminuía la frecuencia de aparición de IAM y del total de afecciones C-V. En los diabéticos, el resultado era más significativo, pues con PA de 120/80 mmHg se reducía la incidencia de $\mathrm{AVE}^{9}$. Nuestro grupo también observó que era posible optimizar la reducción de la PA con la terapia apropiada, pero a pesar de ello, a los 26 años de seguimiento, sólo pudimos demostrar una disminución significativa de la mortalidad, pero no de la incidencia de las complicaciones $\mathrm{C}-\mathrm{V}$ clásicas ${ }^{10,11}$.

En busca de otra explicación para el hallazgo que la reducción de la PA con fármacos no era suficiente para disminuir las complicaciones, se estableció como hipótesis que la presencia permanente de factores asociados, presuntamente causales, denominados clásicamente "factores de riesgo" (FR), podría ser la base del fracaso terapéutico. Si ambos factores, PA y presencia de FR, intervenían en la génesis de las complicaciones y de la mortalidad, el objetivo del tratamiento debía tener como meta principal la reducción de la morbi-mortalidad y no la reducción o normalización de la PA. Desde el Consenso 5 se estableció que la meta del tratamiento debía ser la reducción de la morbi-mortalidad ${ }^{12}$. Conjuntamente se postuló que un mejor manejo y control de los FR conocidos podría mejorar el pronóstico y acentuar la reducción de la $\mathrm{PA}^{12,13}$.

\section{Factores de riesgo}

Los factores de riesgo de la hipertensión arterial eran conocidos desde la década de 1970-79, siendo los principales hipercolesterolemia, hábito tabáquico, diabetes, obesidad, resistencia a la insulina y sedentarismo o mejor, falta de ejercicio físico ${ }^{13-18}$. El control y manejo de esos factores se incorporó a la terapia integral de la HTA como tratamiento "no farmacológico" en los Consensos $5^{\circ}$ y siguientes $^{12,19,20}$. Fue importante entonces determinar si un mejor manejo y control de ellos podría influir en el pronóstico, reduciendo las complicaciones y la mortalidad.

Nuestro grupo, en hipertensos tratados y seguidos 25 años, demostró que la presencia de uno o más factores de riesgo determinaba un peor pronóstico, y que tenían mayor riesgo de complicaciones y muerte los pacientes que presentaban 3 o más de ellos ${ }^{20}$. Además la preexistencia de complicaciones ya establecidas en los órganos blanco condicionaba peor pronóstico. En esos estudios corroboramos que los factores de riesgo presentes en nuestra población eran similares a los comunicados internacionalmente por el JNC $70^{20}$, y también que eran similares a los comunicados clásicamente para la enfermedad coronaria y el accidente cerebro vascular ${ }^{22}$.

Posteriormente, en una cohorte de hipertensos seguidos 30 años, pudimos demostrar que actuando sobre los FR, incluida la PA como uno de ellos, se obtenía una reducción de la morbi-mortalidad, proporcional a la magnitud del cambio de ellos ${ }^{23}$.

En consecuencia, junto al tratamiento farmacológico, es fundamental el manejo integral de todos los factores de riesgo, particularmente los factores de riesgo clásicos, además de la reducción del consumo de sal y la mantención de niveles adecuados de $\mathrm{K}^{24,25}$.

\section{Daño de órganos blanco}

La segunda hipótesis planteaba que la terapia se instala tardíamente y no logra prevenir o detener el avance de la enfermedad. Apoyaba esta presunción el conocimiento muy antiguo que la evolución natural de la enfermedad alcanzaba 12 a 20 años en forma subclínica, antes de la aparición de las complicaciones y que igual evolución se producía aun en los casos en que la hipertensión hubiese sido tratada ${ }^{25}$. Además, en el seguimiento de nuestra cohorte de más de 1.300 hipertensos tratados, comprobamos que al ingreso, la presen- 
cia de daño de los órganos blanco existía en cerca de $30 \%$ de los casos y que constituía un factor de riesgo significativo ${ }^{20}$. El daño estaba representado por las enfermedades ateroescleróticas: IAM, AVE, ateroesclerosis renal, aórtica y de miembros inferiores. Todas ellas se presentaban con una frecuencia diferente, pero con predominio discreto del IAM sobre el AVE E $^{1,20,25}$.

Consecuentemente, la literatura concluyó que no basta con reducir las cifras de PA sino que lo fundamental es prevenir el daño de los órganos blanco y evitar las complicaciones derivadas de ello ${ }^{26}$.

Los estudios fisiopatológicos posteriores, al interiorizarse de la patogenia de la enfermedad ateroesclerótica o aterogénesis, comprobaron que la alteración fisiopatológica inicial y la lesión resultante tenían su raíz en el endotelio vascular, que sufría diversos cambios inflamatorios e histoquímicos que llevaban a la aterotrombosis, con obstrucción vascular, hemorragia o ambos ${ }^{27}$. Simultáneamente la investigación sobre las alteraciones iniciales de la hipertensión arterial, reveló que algunas sustancias vasoactivas del endotelio tenían funciones vasodilatadores (NO) y otras, vasoconstrictoras (endotelina). Se sabe que ellas gatillan el daño vascular, en conjunto con otros factores deletéreos, como radicales libres, citokinas y cambios protrombóticos sanguíneos (alza del hematocrito, alteración de la permeabilidad plaquetaria y aumento de la viscosidad sanguínea) ${ }^{30}$.

Ambos procesos, tanto el observado en hipertensos como el demostrado en pacientes con ateroesclerosis, son fisiopatológicamente similares y podrían constituir una misma enfermedad vascular, susceptible de evolucionar hacia las complicaciones conocidas y a la muerte ${ }^{27,28}$.

Con estos antecedentes, la elevación de la presión arterial comenzó a ser cuestionada como un primum movens de la enfermedad vascular sistémica. Tal vez era un mero indicador, la punta del iceberg ${ }^{29}$.

Tal presunción fue apoyada y reforzada con los resultados del estudio HOT, que reveló que aumentando la reducción de la PA mediante optimización de la terapia hasta conseguir cifras menores a 140/90 $\mathrm{mmHg}$, el riesgo de complicaciones $\mathrm{C}-\mathrm{V}$ se reducía significativamente, en especial respecto al infarto miocárdico y al total de complicaciones C-V. En los hipertensos diabéticos, la reducción a 120/80 mmHg también disminuía el riesgo de $\mathrm{AVE}^{9}$.
Nació así el concepto de pre-hipertensión, pues PA consideradas normales (cifras entre 130-139 sistólica y 85-89 diastólica), presentaban riesgo definido $\mathrm{C}-\mathrm{V}$ con presencia de alteraciones iniciales en los órganos blanco como hipertrofia miocárdica, aumento del espesor de la pared arterial, microalbuminuria y otros, que han sido denominados bio-marcadores del proceso patológico ${ }^{20,30,31}$. Esta concepción fue acogida por el Consenso $7^{\circ}$ de $2003^{20}$.

Pocos años antes, en 1998, uno de nosotros planteaba, en base a los antecedentes conocidos hasta entonces, una concepción integradora, estableciendo la hipótesis de una enfermedad única que comprometía todo el sistema arterial, que podría denominarse "enfermedad cardiovascular", de la cual "la presión arterial sería sólo un epifenómeno". La elevación de la PA "progresaría en paralelo con el proceso histopatológico ateroesclerótico, el que evolucionaría en forma subterránea, dañando las arterias de los órganos blanco para eclosionar repentinamente con alguna de las complicaciones potencialmente letales como el IAM, el AVE y otras menos frecuentes" 29 .

Por los estudios señalados, la PA elevada podría ser considerada sólo como un biomarcador del compromiso lesional progresivo de las paredes arteriales y arteriolares ${ }^{29,32}$. Además, en el adulto mayor, la hipertensión arterial es preferentemente sistólica por incremento de la rigidez aórtica y de la reflexión de la onda de pulso ${ }^{29,32}$. Incluso se ha llegado a redefinir la hipertensión arterial como un síndrome cardiovascular progresivo de etiología compleja ${ }^{32}$.

\section{Cambio de paradigma}

Los antecedentes expuestos sugieren fuertemente que la hipertensión arterial no es una enfermedad por si misma, sino un bio-marcador de una enfermedad subyacente que afecta en forma sistémica a los vasos arteriales del organismo provocando ateroesclerosis y su secuela de complicaciones conocidas. La importancia de la hipertensión residiría en constituir un FR más, que debe integrarse a los ya conocidos para la enfermedad vascular ateroesclerótica. Además, como marcador de un proceso evolutivo subyacente, la magnitud de su elevación y su comportamiento a través del tiempo deben valorarse como factor pronóstico y de eficacia del tratamiento de la afección de base.

Si la PA no es el elemento definitorio de la afec- 
ción vascular y su curva de elevación no reconoce una cifra de corte entre normalidad y enfermedad, parece necesario cambiar el concepto numérico de elevación de la PA por otra dimensión que muestre la curva de su evolución en el tiempo. Así se podría evaluar la importancia pronóstica de elevaciones transitorias, permanentes o de ascenso progresivo a niveles moderados o marcados. De acuerdo a esta concepción, los grados o etapas establecidos por los Consensos del $5^{\circ}$ al $7^{\circ}$, en base a cifras de PA, podrían ser reemplazados por la forma y magnitud de la elevación de la PA en el tiempo. Esta visión permitiría, por un lado, tener una mirada más precoz de la presencia de la enfermedad de base, y por otro, regular la intensidad del tratamiento de acuerdo a la forma de evolucionar de este marcador de riesgo, como lo han sugerido Giles y $\mathrm{col}^{32}$.

En este nuevo paradigma, el factor básico que condiciona el reconocimiento y el pronóstico de la enfermedad arterial pasa a ser el compromiso lesional de los órganos blanco, desde sus etapas iniciales hasta el daño significativo clásico constituido por el IAM, el AVE, la trombosis arterial periférica y las insuficiencias cardíaca, cerebral y renal.

Por tanto, en el ciclo evolutivo de la enfermedad vascular sistémica, se debería considerar una primera etapa sin daño vascular o de real normalidad, con presión arterial igual o inferior a 120/80 $\mathrm{mmHg}$. Esta sería seguida por una segunda etapa, caracterizada por lesiones iniciales de los órganos blanco, que evoluciona en forma asintomática o subclínica, tal como ha sido reconocido por diversos autores ${ }^{8,19,23,32}$ (Tabla 1). Además, Giles y col, han definido otras dos etapas, que relacionan la forma de evolucionar de la elevación de la PA en el tiempo con el número de FR, la aparición de daño orgánico y la presencia clínica de las complicaciones conocidas ${ }^{22,30,32}$ (Tabla 2).

Se configura así un nuevo paradigma clínico para el manejo de la hipertensión arterial, que permite establecer el pronóstico, cualquiera sea el nivel de elevación de la PA. Estaría basado en el espectro de FR y del daño, inicial o avanzado, de los órganos blanco. Así, en conjunto con la evolución de la hipertensión arterial en el tiempo, se puede evaluar mejor el riesgo de desarrollar futuros eventos $\mathrm{C}-\mathrm{V}$. Con este nuevo paradigma se han definido 4 etapas en la evolución natural de la enfermedad vascular sistémica, que se muestran en la Tabla $2^{32}$.

\section{Tabla 1. Alteraciones iniciales de los órganos blanco asociadas a la hipertensión arterial}

\begin{tabular}{|c|c|}
\hline Órganos & Alteración \\
\hline \multirow[t]{4}{*}{ Pr. Arterial } & Pérdida del dip nocturno \\
\hline & Respuesta exagerada al ejercicio \\
\hline & Aumento de la presión de pulso \\
\hline & Sal sensible \\
\hline \multirow[t]{2}{*}{ Corazón } & Hipertrofia septal leve a moderada \\
\hline & Aumento de la presión de llene de la Al \\
\hline \multirow[t]{4}{*}{ Arterias } & Endurecimiento arterial \\
\hline & Aumento presión sistólica \\
\hline & Aumento del grosor de paredes carotídeas \\
\hline & Disfunción endotelial \\
\hline \multirow[t]{2}{*}{ Riñón } & Microalbuminuria \\
\hline & Elevación de la creatinemia \\
\hline \multirow[t]{2}{*}{ Retina } & Espasmo arteriolar \\
\hline & Cruces arteriovenosos \\
\hline
\end{tabular}

Tabla 2. Clasificación de la hipertensión según "hypertension writing group"

\begin{tabular}{|lllll|}
\hline Etapas & Normal & Etapa I & Etapa II & Etapa III \\
Presión arterial & Normal & Elevación intermitente & Elevación mantenida & Elevación marcada \\
Factores riesgo & Ninguno & 1 o más & Varios & Múltiples \\
$\begin{array}{l}\text { Alter. Iniciales órganos } \\
\text { blanco }\end{array}$ & Ninguna & $0-1$ & Más de 2 & Más de 2 y enf. C-V \\
$\begin{array}{l}\text { Complicaciones órganos } \\
\text { blanco }\end{array}$ & Ninguna & Ninguna & Enfermedad progresiva & Eventos definidos \\
\hline
\end{tabular}

Según HWG. Giles y col, J Clin Hypertrens 2005; 7: 505². 
La hipertensión arterial, ¿Sólo un biomarcador de la enfermedad ateroesclerótica sistémica? - O. Román A. et al

De acuerdo a esta concepción, la hipertensión, en cuanto a su magnitud y evolución temporal, pasa a ser solamente un biomarcador de la enfermedad vascular, útil para su detección en etapas tempranas y para guiar el tratamiento y pronóstico en las etapas intermedias o tardías.

Este nuevo paradigma clínico ofrece un enfoque basado en los FR, incluida la hipertensión, para identificar aquellos individuos que tienen una probabilidad razonable de desarrollar eventos CV. Ello permite reclasificar a aquellos sujetos descritos como pre-hipertensos como verdaderamente normales o portadores de la etapa 1 de la enfermedad vascular ${ }^{32}$.

Se puede plantear así una nueva definición de la hipertensión como una alteración cardiovascular compleja que se asocia a niveles elevados de la presión arterial, pero que no es definida sólo por la PA elevada sino por las alteraciones iniciales de los órganos blanco y los FR presentes. Se reconoce así que la alteración de base es una enfermedad vascular sistémica que puede ser denominada "enfermedad sistémica ateroesclerótica". De esta forma, clínicamente se puede indicar el tratamiento a sujetos con gran riesgo (presencia de FR y daño inicial) con presiones inferiores a 140/90. Este es el caso de pre-hipertensos y de hipertensos que tienen diabetes o enfermedad renal.

Este nuevo enfoque de la hipertensión arterial, requiere mayor investigación y opiniones de los especialistas para validar su exactitud y utilidad en la práctica clínica de la enfermedad más prevalerte y que mayor discapacidad y mortalidad determina entre nosotros y en el mundo.

\section{Referencias}

1. Román O, Cuevas G, Martínez D, Cumsille F. Morbimortalidad de la hipertensión tratada en Chile: seguimiento a 15 años. Rev Med Chile 1991; 119: 744-52.

2. Hypertension detection and follow up program cooperative group. The effect of treatment on mortality in mild hypertension: results of the Hypertension, Detection and Follow up Program. N Engl J Med 1982; 307: 97680.

3. Memorandum from the WHO/ISH. 1986. Guidelines for the treatment of high blood pressure. Arch Intern Med 1984; 144: 1045-57.

4. The 1988 Joint National Committee. The 1988 report of the joint national committee on detection, evaluation and treatment of high blood pressure. Arch Intern Med 1988; 148: 1023-38.

5. Hamilton M, Thompson MEN, Wisnieswky TKN. The role of blood pressure control in preventing complications of hypertension. Lancet 1964; 1: 235-38.

6. Veterans Administration Cooperative Study Group on Antihypertensive Agents II. Effects of treatment on morbidity in hypertension. Results in patients with diastolic blood pressure averaging 90 trough $114 \mathrm{mmHg}$. JAMA 1970; 213: 1143-52.

7. Australian Therapeutic Trial in Mild Hypertension. Report by the management committee. Lancet 1980; i: 1261-67.

8. Macmahon S, Peto R, Cutler J. Blood pressure, stroke and coronary heart disease. Part 1. Prolonged differences in blood pressure: prospective observational studies corrected for the regression dilution bias. Lancet 1990; 335 : 765-73.

9. Hansson L, Zanchetti A, Carruthers SG, Dahlof B, Elmfeldt $\mathrm{D}$, Julius $\mathrm{S}$, et al. Effects of intensive blood pressure lowering and low dose aspirin in patients with hypertension: principal results of the Hypertension Optimal Treatment (HOT), randomized trial. Lancet 1998; 351: 1755-62.

10. Román O, Valenzuela M A, Baez D, Herrera E. Optimización de la reducción de presión arterial en hipertensos esenciales. Rev Med Chile 2002; 130: 519-26.

11. Román O, Valenzuela M A, Badilla M, Cumsille F, Rodríguez N. Morbi-mortalidad de la hipertensión esencial tratada. Seguimiento a 26 años. Rev Med Chile 2002; 130: 379-86.

12. Joint National Committee on Detection. Evaluation and treatment of high blood pressure. The fifth report of the Joint National Committee on Detection, Evaluation and treatment of high blood pressure. Arch Intern Med 1993; 153: 154-93.

13. Castelli WP, Anderson K. A population at risk. Prevalence of high cholesterol levels in hypertensive patients in the Framingham study. Am J Med 1986; 80 (Suppl 2A): 23-32.

14. Pooling Project Research Group. Relationship of blood pressure, serum cholesterol, smoking habit, relative weight and ECG abnormalities of major coronary events. Final report of the Pooling Project Research Group J Chronic Dis 1975; 31: 201-306.

15. Helen B, Hubert MPH, Feinleib M, Mc Namara PM, Castelli WP. Obesity as an independent risk factor for cardiovascular disease. A 26 year follow up of participants in the Framingham heart study. Circulation 1983; 67: 968-76.

16. Paffenbarger RS, Hyde RT, Wing AL, Lee IM, Jung DL, 
Kampert JB. The association of change in physical activity level and other life style characteristics with mortality among men. N Engl J Med 1993; 328: 533-7.

17. Saunders J. Alcohol: an important cause of hypertension. Br Med J 1985; 291: 97-104

18. Ferrannini E, Buzzigoli G, Bonadonna R, Giorico M A, Oleggini $\mathrm{M}$, et al. Insulin resistance in essential hypertension. N Engl J Med 1987; 317: 350-7.

19. Joint National Committee on Prevention, Detection, Evaluation and Treatment Of High Blood Pressure. The sixth report of the JNC. Arch Intern Med 1997; 157: 2413-46.

20. Chobanian AV, Bakris GL, Black HR. The seventh report of the Joint National Committee on prevention, detection, evaluation and treatment of high blood pressure: the JNC-7 report. JAMA 2003: 289: 2560-722.

21. Román $\mathrm{O}$, Cuevas G, Bunout D. Influencia de factores de riesgo y terapia farmacológica en la mortalidad de hipertensos esenciales. Rev Med Chile 1998; 126: 745-52.

22. Lanas F. Epidemiología de la cardiopatía coronaria y sus factores de riesgo. En: Enfermedades del Corazón y de los vasos. 2000. Ed. Mediterráneo. Santiago. Chile.

23. Román O, Badilla M, Cuevas G, Valenzuela MA, Barahona S, Báez D, et al. Morbi-mortalidad de la hipertensión arterial según magnitud del cambio de los factores de riesgo. Seguimiento a 30 años. Rev Ch Cardiol 2005; 24 : 11.
24. Stamler J, Rose J, Stamler R, et al. INTERSALT study findings. Public health and medical care implications. Hypertension 1989; 14: 570-7.

25. Kaplan N M. Clinical Hypertension. Sixh Ed. 1994. Williams \& Wilkins. Baltimore USA.

26. Román O, Meza N. Arterial hypertension: end organ damage and its prevention. Intercontinental Cardiology 1996; 5: 49-56.

27. Gimbrone MA, Resnick N, Topper JT. Vascular endothelium, hemodynamics and atherogenesis. In: Vascular Endothelium. 1997, Ed. Schattauer. Stuttgar. págs 28797.

28. Scholkens BA, Unger T. ACE inhibitors, endothelial function and aterosclerosis. 1993. Hoechst Medica Publications Frankfurt.

29. Román O. La enfermedad cardiovascular ¿una nueva entidad? Relación de la hipertensión arterial con ella. Bol Soc Ch Hipertens 1998; 7: 3-5.

30. Román O, Basso B. Valenzuela MA. Cambios estructurales en la hipertensión arterial: regresión con la terapia. Seguimiento a 12 años. Rev Chil Cardiol 1990; 9: 5-15.

31. Ritz E. Renal dysfunction as a novel risk factor: Microalbuminuria and cardiovascular risk. Kidney International 2005; 67 (Supp 93): S25-S28.

32. Giles TD, Berk BC, Black HR, Cohn JR, Kostis JB, Izzo JL, et al. Expanding the definition and classification of hypertension. J Clin Hypertens 2005; 7: 505-12. 